\title{
V4: Pflege und Betreuung von Patienten mit der Nebendiagnose Demenz im Akutkrankenhaus - aktuelle Problemstellungen und erste Lösungsstrategien aus der Pflegepraxis
}

\author{
Eva Faul · Julia Zottl · Hanna Mayer
}

Online publiziert: 30. Oktober 2013

(C) Springer-Verlag Wien 2013

Hintergrund: Im Rahmen des Pilotprojektes DEMAKUT wurde die aktuelle Versorgungs- und Betreuungssituation Demenzerkrankter im Akutkrankenhaus aus der Sicht professionell Pflegender untersucht. Die Pflegepraxis stellt eine wichtige Quelle zur Identifizierung und Benennung spezifischer Pflegesituationen und Problemlagen dar. Des Weiteren existieren bislang kaum Untersuchungen bzgl. der Strategien von Pflegenden, welche aufgrund der alltäglichen Pflegepraxis mit dieser Patientengruppe entwickelt wurden. Dies stellt eine Forschungslücke dar, ist es doch die Pflegepraxis selbst, welche seit längerem gezwungen ist, effiziente Lösungen bzw. wirksame Strategien zur Bewältigung der aktuellen Versorgungssituation zu generieren.

Fragestellung: Die Hauptfragestellungen lauteten:

Welche demenzassoziierten Versorgungs- und Betreuungsprobleme im Akutkrankenhaus gibt es aus der Sicht von Pflegenden?

Welche Strategien werden von den Pflegenden aufgrund der aktuellen Versorgungs- und Betreuungssituation im Akutkrankenhaus bei demenzerkrankten Patienten angewandt?
Methode: Die Pilotstudie folgte einem qualitativen Forschungsansatz und wurde multizentrisch, an vier Krankenanstalten in Wien (Österreich) durchgeführt. Insgesamt wurden 9 Fokusgruppen (mit insgesamt 57 Pflegenden) abgehalten. Die Auswertung erfolgte mit der zusammenfassenden Inhaltsanalyse nach Mayring.

Ergebnisse: Als Resultat der Untersuchung konnten sieben praxisrelevante Problembereiche identifiziert und detailliert beschrieben werden. Ebenso gelang es, entsprechende Strategien aus der alltäglichen Pflegepraxis, wie sie aufgrund der aktuellen Versorgungssituation von den Pflegenden angewandt werden, zu eruieren.

Schlussfolgerung: Die Erkenntnisse des Pilotprojektes DEMAKUT bieten die Möglichkeit, die aktuelle Datenlage um den Bereich der gängigen Pflegepraxis zu erweitern. Pflegenden wird somit die Möglichkeit geboten, einen entscheidenden Beitrag zur Auffindung institutionsspezifischer Versorgungsprobleme zu leisten. Darüber hinaus können erste, aus der Praxis stammende Lösungsansätze neue Wege in der Versorgung Demenzerkrankter im Akutbereich aufzeigen.
E. Faul $(\triangle) \cdot$ J. Zottl $\cdot$ H. Mayer

Institut für Pflegewissenschaft, Universität Wien - Fakultät für

Sozialwissenschaften, Wien, Österreich

E-mail: eva.faul@univie.ac.at 